\title{
Measure on Time Scales with Mathematica
}

\author{
Ünal Ufuktepe and Ahmet Yantır \\ Izmir Institute of Technology and Yasar University \\ Izmir, Turkey \\ unalufuktepe@iyte.edu.tr, ahmet.yantir@yasar.edu.tr
}

\begin{abstract}
In this paper we study the Lebesgue $\Delta$-measure on time scales. We refer to 3 4 for the main notions and facts from the general measure and Lebesgue $\Delta$ integral theory. The objective of this paper is to show how the main concepts of Mathematica can be applied to fundamentals of Lebesgue $\Delta$ - and Lebesgue $\nabla$ - measure on an arbitrary time scale and also on a discrete time scale whose rule is given by the reader. As the time scale theory is investigated in two parts, by means of $\sigma$ and $\rho$ operators, we named the measures on time scales by the set function DMeasure and NMeasure respectively for arbitrary time scales.
\end{abstract}

\section{Introduction}

Time Scales works can be found in 1, 215. The software Mathematica is one of the most powerful tool in discrete and continuous analysis. Computational works on time scale calculus are collected in TimeScale package [6. As probability theory is established on continuous and discrete analysis, we generalize TimeScale package in order to calculate the measure on time scales as a first step of probability theory and statistics.

In this paper first we give an introduction to the time scales, and we present the connection of the Lebesgue measure with the Lebesgue $\Delta$-measure on an arbitrary bounded time scale $\mathrm{T}$ such that $\min \mathrm{T}=a$ and $\max \mathrm{T}=b$. We set out many basic concepts from measure theory to the $\Delta$-measurable space. Finally we use Mathematica to illustrate the Lebesgue $\Delta$ - and $\nabla$ - measures to set out the differences between Lebesgue measure and Lebesgue $\Delta(\nabla)$ - measure.

\section{$2 \Delta$ - and $\nabla$ - Measure on Time Scale}

Let $\mathrm{T}$ be a time scale, $a<b$ be points in $\mathrm{T}$, and $[a, b)$ be half closed bounded interval in T, $\sigma$ and $\rho$ be the forward and backward jump operators respectively on T. Let

$$
\Im_{1}=\left\{\left[a^{\prime}, b^{\prime}\right) \bigcap \mathrm{T}: a^{\prime}, b^{\prime} \in \mathrm{T}, a^{\prime} \leq b^{\prime}\right\}
$$

be the family of all left closed and right open intervals of T. Then $\Im_{1}$ is a semiring. Here $\left[a^{\prime}, a^{\prime}\right)=\varnothing \cdot m_{1}: \Im_{1} \rightarrow[0, \infty]$ is a set function which assigns to each interval its length: $m_{1}\left(\left[a^{\prime}, b^{\prime}\right)\right)=b^{\prime}-a^{\prime}$. So if $\left\{I_{n}\right\}$ is a sequence of disjoint intervals in $\Im_{1}$ then $m_{1}\left(\bigcup I_{n}\right)=\sum m_{1}\left(I_{n}\right)$. 
Let $E \subset \mathrm{T}$. By Carathéodory extension, outer measure of $E$ is

$$
m_{1}^{*}(E)=\inf _{E \subset \bigcup_{n} I_{n}} \sum m_{1}\left(I_{n}\right)
$$

where $I_{n} \in \Im_{1}$. If there is no such covering of $E$, then $m_{1}^{*}(E)=\infty$.

Definition 1. A set $E \subset \mathrm{T}$ is said to be $\Delta$-measurable if for each set $A$

$$
m_{1}^{*}(A)=m_{1}^{*}(A \cap E)+m_{1}^{*}\left(A \bigcap E^{c}\right)
$$

where $E^{c}=\mathrm{T}-E$. If $E$ is $\Delta$-measurable then $E^{c}$ is also $\Delta$-measurable. Clearly $\varnothing$ and $\mathrm{T}$ are $\Delta$ - measurable.

Let $\mathfrak{M}\left(m_{1}^{*}\right)=\{E \subset \mathrm{T}: E$ is $\Delta$ measurable $\}$ be a family of $\Delta$-measurable sets.

Corollary 1. $\mathfrak{M}\left(m_{1}^{*}\right)$ is a $\sigma$ algebra.

Definition 2. The restriction of $m_{1}^{*}$ to $\mathfrak{M}\left(m_{1}^{*}\right)$ is called Lebesgue $\Delta$ - measure and denoted by $\mu_{\Delta}$.

So $m_{1}^{*}(E)=\mu_{\Delta}(E)$ if $E \in \mathfrak{M}\left(m_{1}^{*}\right)$.

Similarly, if we take $\mathfrak{F}_{2}=\left\{\left(a^{\prime}, b^{\prime}\right]: a^{\prime}, b^{\prime} \in \mathrm{T}, a^{\prime} \leq b^{\prime}\right\}$ where $\left(a^{\prime}, a^{\prime}\right]$ is understood as an empty set then $m_{2}: \mathfrak{F}_{2} \rightarrow[0, \infty]$ such that $m_{2}\left(\left(a^{\prime}, b^{\prime}\right]\right)=b^{\prime}-a^{\prime}$ is a countably additive measure. Then $\mathfrak{M}\left(m_{2}^{*}\right)$ is the set of $\nabla$ - measurable sets and $\mu_{\nabla}$ is Lebesgue $\nabla$ - measure on $\mathrm{T}$.

Proposition 1. Let $\left\{E_{n}\right\}$ be an infinite decreasing sequence of $\Delta$-measurable sets, that $i s$, a sequence $E_{1} \supset E_{2} \supset \cdots \supset E_{n} \supset \cdots, E_{i} \in \mathfrak{F}_{1}$ for each $i, \cap E_{i} \in \mathfrak{F}_{1}$ and $m_{1}^{*}\left(E_{1}\right)<\infty$. Then

$$
m_{1}^{*}\left(\bigcap_{n=1}^{\infty} E_{i}\right)=\lim _{n \rightarrow \infty} m_{1}^{*}\left(E_{n}\right)
$$

\section{$3 \quad \nabla$ and $\Delta$ Measures with Mathematica}

Theorem 1. For each $t_{0} \in \mathrm{T}-\{\min \mathrm{T}\}$ the $\nabla$-measure of the single point set $\left\{t_{0}\right\}$ is given by $\mu_{\nabla}\left(\left\{t_{0}\right\}\right)=t_{0}-\rho\left(t_{0}\right)$.

Proof. Case 1. Let $t_{0}$ be left scattered. Then $\left\{t_{0}\right\}=\left(\rho\left(t_{0}\right), t_{0}\right] \in \mathfrak{F}_{2}$. So $\left\{t_{0}\right\}$ is $\nabla$ measurable and $\mu_{\nabla}\left(\left\{t_{0}\right\}\right)=t_{0}-\rho\left(t_{0}\right)$.

Case 2. Let $t_{0}$ be left dense. Then there exists an increasing sequence $\left\{t_{k}\right\}$ of points of $\mathrm{T}$ such that $t_{k} \leq t_{0}$ and $t_{k} \uparrow t_{0}$. Since $\left\{t_{0}\right\}=\bigcap_{k=1}^{\infty}\left(t_{k}, t_{0}\right] \in \mathfrak{F}_{2}$. Therefore $\left\{t_{0}\right\}$ is $\nabla$ measurable. By continuity

$$
\mu_{\nabla}\left(\left\{t_{0}\right\}\right)=\mu_{\nabla}\left(\bigcap_{k=1}^{\infty}\left(t_{k}, t_{0}\right]\right)=\lim _{n \rightarrow \infty} \mu_{\nabla}\left(\left(t_{n}, t_{n}\right]\right)=\lim _{n \rightarrow \infty} t_{0}-t_{n}=0
$$

which is the desired result since $t_{0}$ is left dense. 
Theorem 2. If $a, b \in \mathrm{T}$ and $a \leq b$ then 1) $\left.\mu_{\nabla}((a, b])=b-a, 2\right) \mu_{\nabla}((a, b))=$ $\rho(b)-a, 3)$ If $a, b \in \mathrm{T}-\min \mathrm{T}$ then $\mu_{\nabla}([a, b))=\rho(b)-\rho(a)$ and $\mu_{\nabla}([a, b])=$ $b-\rho(a)$.

Proof. From the definition $\mu_{\nabla}((a, b])=b-a$.

$$
\begin{gathered}
\mu_{\nabla}((a, b])=\mu_{\nabla}((a, b) \bigcup\{b\})=\mu_{\nabla}((a, b))+\mu_{\nabla}(\{b\})=\mu_{\nabla}((a, b))+b-\rho(b) \\
b-a=\mu_{\nabla}((a, b))+b-\rho(b)
\end{gathered}
$$

So $\mu_{\nabla}((a, b))=\rho(b)-a$.

iii) Let $a, b \in \mathrm{T}-\min \mathrm{T}$.

$\mu_{\nabla}([a, b))=\mu_{\nabla}(\{a\} \bigcup(a, b))=\mu_{\nabla}(\{a\})+\mu_{\nabla}((a, b))=a-\rho(a)+\rho(b)-a=\rho(b)-\rho(a)$ $\mu_{\nabla}([a, b])=\mu_{\nabla}([a, b) \bigcup\{b\})=\mu_{\nabla}([a, b))+\mu_{\nabla}(\{b\})=\rho(b)-\rho(a)+b-\rho(b)=b-\rho(a)$

Theorem 3. For each $t_{0} \in \mathrm{T}-\{\max \mathrm{T}\}$ the single point set $\left\{t_{0}\right\}$ is $\Delta$-measurable and its $\Delta$-measure is given by $\mu_{\Delta}\left(\left\{t_{0}\right\}\right)=\sigma\left(t_{0}\right)-t_{0}$.

Proof. Case 1. Let $t_{0}$ be right scattered. Then $\left\{t_{0}\right\}=\left[t_{0}, \sigma\left(t_{0}\right)\right) \in \mathfrak{F}_{1}$. So $\left\{t_{0}\right\}$ is $\Delta$ - measurable and $\mu_{\Delta}\left(\left\{t_{0}\right\}\right)=\sigma\left(t_{0}\right)-t_{0}$.

Case 2. Let $t_{0}$ be right dense. Then there exists a decreasing sequence $\left\{t_{k}\right\}$ of points of $\mathrm{T}$ such that $t_{0} \leq t_{k}$ and $t_{k} \downarrow t_{0}$. Since $\left\{t_{0}\right\}=\bigcap_{k=1}^{\infty}\left[t_{0}, t_{k}\right) \in \mathfrak{F}_{1}$. Therefore $\left\{t_{0}\right\}$ is $\Delta$ - measurable. By proposition 1

$$
\mu_{\Delta}\left(\left\{t_{0}\right\}\right)=\mu_{\Delta}\left(\bigcap_{k=1}^{\infty}\left[t_{0}, t_{k}\right)\right)=\lim _{n \rightarrow \infty} \mu_{\Delta}\left(\left[t_{0}, t_{n}\right)\right)=\lim _{n \rightarrow \infty} t_{n}-t_{0}=0
$$

which is the desired result since $t_{0}$ is right dense.

Every kind of interval can be obtained from an interval of the form $[a, b)$ by adding or subtracting the end points $a$ and $b$. Then each interval of $\mathrm{T}$ is $\Delta$ measurable.

Theorem 4. If $a, b \in \mathrm{T}$ and $a \leq b$ then

1) $\left.\mu_{\Delta}([a, b))=b-a, 2\right) \mu_{\Delta}((a, b))=b-\sigma(a)$, 3) If $a, b \in \mathrm{T}-\max \mathrm{T}$ then $\mu_{\Delta}((a, b])=\sigma(b)-\sigma(a)$ and $\mu_{\Delta}([a, b])=\sigma(b)-a$.

To illustrate these properties with mathematica, our TimeScale package must be loaded.

$\operatorname{In}[1]:=<<$ TimeScale $^{6}$

Let the time scale is as follows

$\operatorname{In}[2]:=T=\{5<=x<=7 \| x==15 / 2|| 9<=x<=11|| x==12|| x==18\}$

We must define set function with respect to the interval or a single point

$\operatorname{In}[3]:=$ ClosedSet $=\{$ closed,a,b,closed $\} ;$ OpenSet $=\{$ open,a,b,open $\}$; 


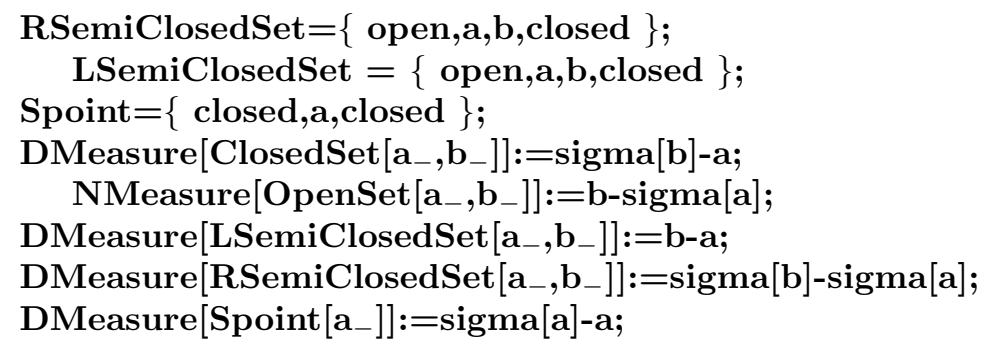

Now, we would like to find the measures of $\{5\}$ and $(5,11] \mathbf{I n}[4]:=$ DMeasure[Spoint [7]]

Out $[4]:=\frac{1}{2}$

$\operatorname{In}[5]:=$ DMeasure[RSemiClosedSet $[5,11]]$

Out $[6]:=7$

Mathematica applications of $\nabla$-measure also can be done as $\Delta$-measure. The sigma operator must be replaced by the $\mathrm{r}$ operator also.

\section{Conclusion}

In this work we worked on Lebesgue $\Delta$-measure and Lebesgue $\nabla$-measure on time scales with Mathematica. We investigated each of these two measures in two parts, arbitrary time scales and discrete time scales. To do these we improved the TimeScale package. In the future, we will work on generalizing the probability theory on Time Scales with Mathematica.

\section{Acknowledgement}

This work is supported by The Scientific \& Technological Research Council of Turkey.

\section{References}

1. Bohner,M.\& Peterson,A., Dynamic Equations on Time Scales, Birkhäuser Boston, 2001.

2. Bohner,M.\& Peterson,A., Advances in Dynamic Equations on Time Scales, Birkhäuser Boston, 2004.

3. Guseinov, G.S., Integration on time scales, J.Math. Anal.Appl. 285, 1, 107-127, (2003).

4. Guseinov, G.S. \& Kaymakalan, B., Basics of Riemann delta and bale integration on time scales, J.Difference Equ. Appl. 8, 11, 1001-1017, (2002).

5. Hilger, S.: Analysis on measure chains-a unified approach to continuous and discrete calculus, 1990, Results Math. 18, 18-56.

6. Yantir, A.\& Ufuktepe Ü., Mathematica Aplications on Time Scales for Calculus, 2005, Lecture Notes in Computer Science, 3482, 529-537. 